\title{
Progressive treadmill cognitive dual-task gait training on the gait ability in patients with chronic stroke
}

\author{
Keun-Jo Kim, Kyung-Hun Kim* \\ Department of Physical Therapy, Gimcheon University, Gimcheon, Korea
}

The purpose of this study was to investigate the effects of progressive treadmill cognitive dual-task training on gait performance in individuals with chronic stroke patients. Twenty-six participants with chronic hemiparesis were recruited to this study and randomly distributed into two groups: progressive treadmill cognitive dual-task gait training (PTCDG group, 13 patients), conventional treadmill gait training (CTG group, 13 patients). All patients underwent 20 sessions of treadmill gait training with a harness ( 5 times per week, for a total of 4 weeks). The GAITRite was used to analyze gait kinematics, and 10-m walking test (10MWT) wear used as the clinical evaluation indicators. After training, PTCDG group showed significant improvement in gait velocity, cadence, single support time, and 10MWT compared to the control group. However there was no significant difference between the groups in the stride lengths in the affected and unaffected sides. The study verified that progressive treadmill cognitive dual-task gait training had a positive influence on the gait and clinical gait index in chronic stroke patients.

Keywords: Cognitive, Dual-task, Gait, Stroke

\section{INTRODUCTION}

Cognitive function refers to the ability to understand things in everyday life, commencing a behavior, planning and thinking an action, and adapting to the environment while resolving an issue. Cognitive domains include concentration, memory, systematization, problem solving, abstraction, and language use (Campbell et al., 1990). After 3 months from the onset of stroke patients, $25 \%$ of patients show dementia and $50 \%-70 \%$ of patients demonstrate partial cognitive dysfunction (Desmond et al., 2000).

Dual-task training requires a subject to perform multiple tasks at the same time and it emphasizes the role of concentration and cognition in posture and gait control (Silsupadol et al., 2006). Various studies have evaluated the interactions between cognitive tasks and motor tasks (Paul et al., 2009).

Studies on dual-tasks were conducted mainly by using cognitive dual-task training design, which evaluates the interaction among posture control, concentration, and cognition by conducting cognitive tasks and intelligence tasks simultaneously such as giving proper answers, adding up figures, and making words while making a gait or standing posture (Bowen et al., 2001).

The cognitive function of patients with stroke patients affects the gait and they must have cognitive abilities that can understand and learn commands for successful rehabilitation. The interference between motor control activities while conducting two tasks (e.g., a cognitive task and gait) simultaneously is an important therapeutic environmental factor for independently improving the function of patients with neurological impairment. Therefore, a therapist must include both cognitive and exercise treatments in the rehabilitation therapy plan (Haggard et al., 2000).

Among recent studies on dual-task with stroke patients and Parkinson disease, Yang et al. (2007b) reported that dual-task gait training influenced the walking of patients with stroke patients positively. O'Shea et al. (2002) showed that applying cognitive tasks to patients with Parkinson disease with decreased motor ability while they were making postures or gait enhanced their cognitive task performance and improved their gait ability. Evans et al. (2009) evaluated the changes in gait according to the du-
${ }^{*}$ Corresponding author: Kyung-Hun Kim (iD https://orcid.org/0000-0002-5889-1948 Department of Physical Therapy, Gimcheon University, 214 Daehak-ro, Gimcheon 39528, Korea

Tel: +82-2-7110-4690, Fax: +82-54-420-4467, E-mail: huni040@naver.com

Received: July 31, 2018 / Accepted: September 11, 2018
This is an Open Access article distributed under the terms of the Creative Commons Attribution Non-Commercial License (http://creativecommons.org/licenses/by-nc/4.0/) which permits unrestricted non-commercial use, distribution, and reproduction in any medium, provided the original work is properly cited. 
al-task training for 19 subjects with stroke patients. They revealed that the dual-task training group significantly improved the dual-task performance abilities in the daily life movements. Dennis et al. (2009) evaluated the velocity of patients with chronic stroke patients while conducting a motor-cognitive task and showed that the gait velocity was maintained while conducting a cognitive task and gait at the same time.

The dual-task training is an important part of the gait training of patient with stroke patients in the task-oriented aspect and it should reflect the motor and cognitive functions in everyday life (Bowen et al., 2001; Melzer et al., 2007). Therefore, the objective of this study was to propose a new rehabilitation protocol for improving the functions of patients with stroke patients by evaluating the changes in the gait ability after applying the progressive treadmill cognitive dual-task gait training to the patients with chronic stroke patients. This study hypothesized that progressive treadmill cognitive dual-task gait training would improve the gait function of participants compared to conventional treadmill gait training.

\section{MATERIALS AND METHODS}

\section{Participant}

This study was carried out with 30 subjects who visited Karis Hospital located in Bundang-gu, Seongnam city, Gyeonggi-do
Province for treatment. The selection criteria were as follows: (a) patients who medically had stroke for at least 6 months, (b) could walk on treadmill for more than $30 \mathrm{~min}$, (c) score on the Korean Mini-Mental Status Examination not lower than 24, (d) ability to walk independently without ant gait aid, and (e) voluntarily signed the study participation consent form before conducting the experiment. This study excluded patients who suffered from a vestibular apparatus or cerebellum associated disease, who had visual or hearing impairment, who were taking medications that could interfere with the gait ability, and who were judged to be inadequate by researchers. All procedures were approved by the Gimcheon University Institutional Review Board (GU-201801-HRa-02-01).

\section{Protocol}

This study used a pretest-posttest control group design. We conducted a pretest to the study subjects who signed the consent form 1 week before commencing the exercise program and a posttest was given to them after completing the 4-week training. The gait ability of these pretest-posttest subjects was measured by using the 10-m gait test (10MWT) and a gait analysis system.

Sample size was calculated using the G*Power 3.1 the alpha levels set at 0.60 and 0.50 , respectively, and the effect size of 0.80 . Thirty subjects who met the selection criteria were randomly divided into two groups to minimize the selection bias. Patients were randomized to the PTCDG group (A) or CTG group (B) by

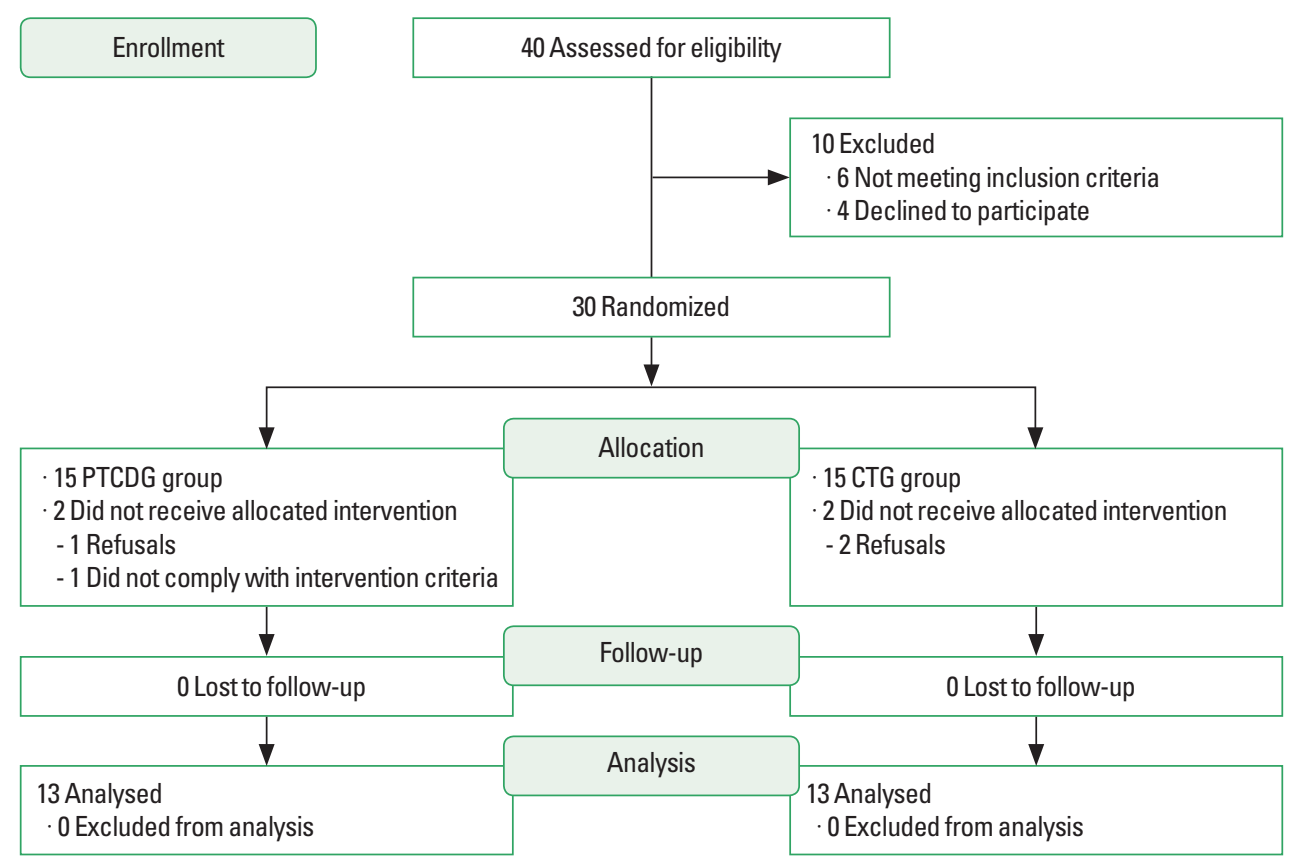

Fig. 1. Flow diagram of the study. 
an independent person who chose one of 30 sealed envelopes 30 min before the start of the intervention, thus randomly allocating 15 to the PTCDG group and 15 to the CTG group. Both groups trained 5 days per week and 30 min per day for 4 weeks. A pretest was given before starting the training and a posttest was given after completing the 4-week training (Fig. 1).

When a subject was not capable of sustaining the training for 30 min due to limited physical ability, the training stopped for the subject. If a subject expressed fatigue, pain, abnormal breathing, or complexion change, the subject took 5-min break (Kesar et al., 2011).

\section{Intervention}

\section{PTCDG group}

The progressive treadmill cognitive dual-task gait training group performed gait on a medical and rehabilitation low-speed treadmill with wearing a harness and progressively conducting cognitive tasks at the same time. The speed of the treadmill was determined by a patient at the appropriate velocity for the patient to walk on the flat surface. When a patient was able to walk for more than 20 sec stably (Yang et al., 2007a), the velocity increased by $0.1 \mathrm{~km} / \mathrm{hr}$ per session (Ditor et al., 2005).

The first-week cognitive tasks included speaking numbers task which asked a subject to speak numbers between 1 and 100 randomly without repeating a number (e.g., 1-26-75-43-98). Color distinction asked a subject to answer as "Red-Yes \& Blue-No" using experimental color plates while gait (Hollman et al., 2007). Counting numbers backward asked a subject to count numbers backward (e.g., 3-2-1 and 9-8-7-6) (Pellecchia, 2005).

The second-week cognitive tasks were arithmetic subtraction (e.g., 100-3 and 100-48), arithmetic addition (e.g., 100+7 and $100+12)$, and word making, creating words using a given consonant (e.g., $\neg-$ 고구마 $=$ seet potato, 과자 $=$ snack, 감자 $=$ porato, 가 위= scissors).

The third-week cognitive tasks were memorizing objects, speaking objects or words, or completing sentences. Remembering objects was to memorize and recite phone numbers, words, addresses, objects, and price (e.g., 010-7640-1234, desk, chair, and $\$ 2$ worth of apple). Speaking objects or words was to say the names of mountains, countries, animals, etc. (e.g., Mountain Mt. Baekdu, Mt. Halla, Mt. Sobaek, and Mt. Gyeryong). Completing sentences was to create diverse sentences using several words.

The fourth-week cognitive tasks asked subjects to say three- to four-syllable words reversely, tell a story to another person with connecting sentences (e.g., telling what the subject did last night), and mimic a sentence, which was to speak a complex sentence harmoniously.

During the 4-week training, these 12 tasks were given as weekly tasks. In the first week, each task was carried out for 5 min for each set and three tasks $(15 \mathrm{~min}$ ) were given for each set. Two sets were given for each day. Two physiotherapists participated in the training. One physiotherapist continuously assigned the task and the other physiotherapist placed his hand on the emergency stop button on the right side of the treadmill to respond to a potential safety accident during training. When a subject showed or expressed fatigue, pain, breathing abnormality, and changes in complexion, the subject took a rest for $5 \mathrm{~min}$.

\section{CTG group}

The conventional treadmill gait training group had gait training at the speed desired by a subject without conducting a cognitive task, with wearing a harness. Subjects in this group conducted a gait training with wearing a suspension device on a treadmill (QUASAR MED, HP COSMOS, Nussdorf-Traunstein, Germany), which provided a $0 \%$ weight support condition. When a patient was able to walk for more than 20 sec stably (Yang et al., 2007a), the velocity increased by $0.1 \mathrm{~km} / \mathrm{hr}$ per session (Ditor et al., 2005).

\section{Measurements}

The 10MWT is an easy and simple method for predicting the gait ability of a patient with stroke patients (Dean et al., 2001). 10MWT was a good method for evaluating the velocity, which reflected movement capability of patients with stroke patients, who had a stroke more than 6 months before, well. This study used a 10MWT and this test presents the velocity in seconds. Each patient completed the 10MWT test 3 times for an average value. The test-retest reliability for the stroke patients high $(r=0.87)$ (Green et al., 2002).

GAITRite (CIR System Inc., Havertown, PA, USA) was used to measure the gait ability. GAITRite is an electric gait panel (length, $5 \mathrm{~m}$; width, $61 \mathrm{~cm}$; and height, $0.6 \mathrm{~cm}$ ). A total of 16,128 sensors are arranged vertically along the gait plate at every $1.27 \mathrm{~cm}$ to measure variables temporally and spatially. Each sensor is $1 \mathrm{~cm}$ in diameter. The equipment collects the load from the subject's feet at a sampling rate of $80 \mathrm{~Hz}$ and sends the information to a computer via the serial interface cable. The information about the collected spatial and temporal variables was processed by the software program. GAITRite allows researchers to measure the temporal gait variables and the spatial gait characteristics of function- 
al gait index accurately through a computerized analysis. Measurements are taken while a subject walks $11 \mathrm{~m}$ at a comfortable velocity. The measurements of the first and last $3 \mathrm{~m}$ are excluded and only the gait variables collected at the middle $5 \mathrm{~m}$ were collected and used for the analysis.

It was measured 3 times and a mean value was used for the study. A subject took a 3-min rest between each measurement to minimize the effects on the muscle fatigue. The measuring person reliability of this test is $r=0.90$, while the correlation coefficient of the comfortable gait measurement 0.96 or higher (van Uden and Besser, 2004).

\section{Statistical analysis}

Data analysis was performed using IBM SPSS Statistics ver. 21.0 (IBM Co., Armonk, NY, USA). The normality of variables was assessed using the Shapiro-Wilk test. Age, height, weight, poststroke, and Mini-Mental State Examination were analyzed through independent $t$-tests. Chi-square testing analyzed the groups for gander, paretic side, and etiology. Paired $t$-test was used for within-group comparison and independent $t$-test was used for between-group comparison. The statistical significance level of all data was set to alpha $=0.05$.

\section{RESULTS}

The demographic characteristics of patients with stroke are presented in Table 1 . There was no statistically significant difference between the groups regarding baseline demographic (Table 1). Table 2 showed significant paired difference in the all variable before and after training. Although insignificant, the percent increase in the mean difference of all the variables was better PTC-
DG group than the CTG group (Tables 2, 3). After training, gait velocity was significantly increased in the PTCDG group than in the CTG group. The change values amount before and after training was $-9.11 \mathrm{~cm} / \mathrm{sec}$ and $-5.08 \mathrm{~cm} / \mathrm{sec}$, respectively. Cadence showed a significant improvement in the PTCDG group (change values, $-9.02 \mathrm{step} / \mathrm{min}$ ) compared with the CTG group (change values, $-4.35 \mathrm{step} / \mathrm{min})$. However, stride lengths in the affected and unaffected sides were not significantly different between the two groups. The single affected single support time and unaffected single support time significantly increased in the PTCDG group (change values, $-6.92 \%,-7.92 \%$, respectively) than in the

Table 1. Demographic characteristics of patients with stroke $(n=26)$

\begin{tabular}{llcc}
\hline Characteristic & Group & \multicolumn{1}{c}{ Value } & P-value \\
\hline Age $(\mathrm{yr})$ & PTCDG & $52.62 \pm 9.84(36-69)$ & 0.392 \\
& CTG & $56.15 \pm 10.82(38-69)$ & \\
Height $(\mathrm{cm})$ & PTCDG & $166.48 \pm 7.34(155-178)$ & 0.658 \\
& CTG & $167.57 \pm 4.81(160-176)$ & \\
Weight $(\mathrm{kg})$ & PTCDG & $63.22 \pm 8.05(51-84)$ & 0.724 \\
& CTG & $64.42 \pm 9.15(52-83)$ & \\
Post-stroke (mo) & PTCDG & $12.62 \pm 3.52(6-19)$ & 0.430 \\
& CTG & $11.46 \pm 3.80(6-18)$ & \\
MMSE & PTCDG & $27.23 \pm 1.69(25-30)$ & 0.680 \\
& CTG & $27.46 \pm 1.05(26-29)$ & \\
Gander, male:female & PTCDG & $8(61.5): 5(38.5)$ & 0.691 \\
& CTG & $7(53.8): 6(46.2)$ & \\
Paretic side, right:left & PTCDG & $8(61.5): 5(38.5)$ & 0.239 \\
& CTG & $5(38.5): 8(61.5)$ & \\
Etiology, infarction:hemorrhage & PTCDG & $7(53.8): 6(46.2)$ & 0.691 \\
& CTG & $8(61.5): 5(38.5)$ & \\
\hline
\end{tabular}

Values are presented as mean \pm standard deviation (range) or number (\%). PTCDG, progressive treadmill cognitive dual task gait training group; CTG, conventional treadmill gait training group; MMSE, mini-mental state examination.

Table 2. Differences in the pretest and posttest progressive treadmill cognitive dual task gait training group

\begin{tabular}{lccccccc}
\hline Variable & Pretest & Posttest & \% Mean difference & $95 \%$ Cl & $d f$ & $T$-value & $P$-value \\
\hline Gait velocity (cm/sec) & $42.37 \pm 10.23$ & $51.48 \pm 10.02$ & -9.11 & -11.08 to -7.13 & 12 & -10.041 & $0.000^{*}$ \\
Cadence (step/min) & $71.44 \pm 11.08$ & $80.46 \pm 11.47$ & -9.02 & -12.31 to -5.74 & 12 & -5.980 & $0.000^{*}$ \\
Affected stride length (cm) & $67.40 \pm 12.23$ & $77.14 \pm 12.93$ & -9.74 & -12.20 to -7.27 & 12 & -8.597 & $0.000^{*}$ \\
Unaffected stride length (cm) & $67.38 \pm 11.98$ & $77.79 \pm 13.22$ & -10.40 & -12.51 to -8.30 & 12 & -10.766 & $0.000^{*}$ \\
Affected single support time (\%) & $21.46 \pm 7.80$ & $28.38 \pm 6.33$ & -6.92 & -8.48 to -5.35 & 12 & -9.612 & $0.000^{*}$ \\
Unaffected single support time (\%) & $30.92 \pm 8.35$ & $38.85 \pm 6.88$ & -7.92 & -10.80 to -5.04 & 12 & -5.990 & $0.000^{*}$ \\
Affected swing phase (\%) & $31.54 \pm 2.95$ & $33.01 \pm 2.28$ & -1.47 & -2.00 to -0.93 & 12 & -5.989 & $0.000^{*}$ \\
Affected stance phase (\%) & $68.26 \pm 2.95$ & $66.99 \pm 2.28$ & 1.47 & 0.93 to 2.00 & 12 & 5.989 & $0.000^{*}$ \\
10-m gait test(m/sec) & $0.73 \pm 0.20$ & $0.89 \pm 0.21$ & -0.16 & -0.20 to 0.11 & 12 & -8.022 & $0.000^{*}$ \\
\hline
\end{tabular}

Values are presented as mean \pm standard deviation.

$\mathrm{Cl}$, confidence interval; $d f$, degree of freedom.

${ }^{*} P<0.05$, statistical significance. 
Table 3. Differences in the pretest and posttest conventional treadmill gait training group

\begin{tabular}{lccccccc}
\hline \multicolumn{1}{c}{$d f$} & Pretest & Posttest & \% Mean difference & $95 \% \mathrm{Cl}$ & $d f$ & T-value & $P$-value \\
\hline Gait velocity (cm/sec) & $44.90 \pm 12.23$ & $49.98 \pm 13.24$ & -5.08 & -7.78 to -2.37 & 12 & -4.091 & $0.001^{*}$ \\
Cadence (step/min) & $69.92 \pm 11.00$ & $74.27 \pm 12.09$ & -4.35 & -7.86 to -0.84 & 12 & -2.698 & $0.019^{*}$ \\
Affected stride length (cm) & $74.19 \pm 15.50$ & $80.59 \pm 12.07$ & -6.40 & -9.25 to -3.54 & 12 & -4.884 & $0.000^{*}$ \\
Unaffected stride length (cm) & $74.33 \pm 15.17$ & $81.22 \pm 11.84$ & -6.89 & -10.20 to -3.59 & 12 & -4.547 & $0.001^{*}$ \\
Affected single support time (\%) & $20.69 \pm 6.07$ & $23.77 \pm 5.72$ & -3.08 & -3.95 to -2.21 & 12 & -7.698 & $0.000^{*}$ \\
Unaffected single support time (\%) & $30.15 \pm 9.03$ & $33.69 \pm 6.56$ & -3.54 & -5.85 to -1.23 & 12 & -3.339 & $0.006^{*}$ \\
Affected swing phase (\%) & $30.72 \pm 3.12$ & $31.28 \pm 3.50$ & -0.55 & -1.21 to 0.10 & 12 & -1.844 & 0.090 \\
Affected stance phase (\%) & $69.27 \pm 3.12$ & $68.72 \pm 3.50$ & 0.55 & -0.10 to 1.21 & 12 & 1.844 & 0.090 \\
10-m gait test (m/sec) & $0.75 \pm 0.20$ & $0.81 \pm 0.22$ & -0.06 & -0.09 to -0.03 & 12 & -4.708 & $0.001^{*}$
\end{tabular}

Values are presented as mean \pm standard deviation.

$\mathrm{Cl}$, confidence interval; $d f$, degree of freedom.

${ }^{*} P<0.05$, statistical significance.

Table 4. Differences in the change values of the progressive cognitive dual task training and conventional treadmill training group

\begin{tabular}{|c|c|c|c|c|c|c|c|c|}
\hline Variable & Group & No. & Change values & \% Mean difference & $95 \% \mathrm{Cl}$ & $d f$ & T-value & $P$-value \\
\hline \multirow[t]{2}{*}{ Gait velocity (cm/sec) } & PTCDG & 13 & $-9.11 \pm 3.27$ & -4.03 & -7.20 to -0.86 & 24 & -2.622 & $0.015^{*}$ \\
\hline & CTG & 13 & $-5.08 \pm 4.47$ & & & & & \\
\hline \multirow[t]{2}{*}{ Cadence (step/min) } & PTCDG & 13 & $-9.02 \pm 5.44$ & -4.68 & -9.23 to -0.12 & 24 & -2.119 & $0.045^{*}$ \\
\hline & CTG & 13 & $-4.35 \pm 5.80$ & & & & & \\
\hline \multirow[t]{2}{*}{ Affected stride length (cm) } & PTCDG & 13 & $-9.74 \pm 4.08$ & -3.34 & -6.91 to 0.24 & 24 & -1.928 & 0.066 \\
\hline & CTG & 13 & $-6.40 \pm 4.72$ & & & & & \\
\hline \multirow[t]{2}{*}{ Unaffected stride length (cm) } & PTCDG & 13 & $-10.40 \pm 3.48$ & -3.51 & -7.22 to 0.20 & 24 & -1.953 & 0.063 \\
\hline & CTG & 13 & $-6.89 \pm 5.47$ & & & & & \\
\hline \multirow[t]{2}{*}{ Affected single support time (\%) } & PTCDG & 13 & $-6.92 \pm 2.60$ & -3.85 & -5.55 to -2.15 & 24 & -4.669 & $0.000^{*}$ \\
\hline & CTG & 13 & $-3.08 \pm 1.44$ & & & & & \\
\hline \multirow[t]{2}{*}{ Unaffected single support time (\%) } & PTCDG & 13 & $-7.92 \pm 4.77$ & -4.38 & -7.88 to $-0,89$ & 24 & -2.587 & $0.016^{*}$ \\
\hline & CTG & 13 & $-3.54 \pm 3.82$ & & & & & \\
\hline \multirow[t]{2}{*}{ Affected swing phase (\%) } & PTCDG & 13 & $-1.47 \pm 0.88$ & -0.91 & -1.72 to -0.12 & 24 & -2.360 & $0.027^{*}$ \\
\hline & CTG & 13 & $-0.55 \pm 1.08$ & & & & & \\
\hline \multirow[t]{2}{*}{ Affected stance phase (\%) } & PTCDG & 13 & $-1.47 \pm 0.88$ & -0.91 & -1.72 to -0.12 & 24 & -2.360 & $0.027^{*}$ \\
\hline & CTG & 13 & $-0.55 \pm 1.08$ & & & & & \\
\hline \multirow[t]{2}{*}{ 10-m gait test (m/sec) } & PTCDG & 13 & $-0.16 \pm 0.07$ & -0.10 & -0.14 to -0.05 & 24 & -4.163 & $0.000^{*}$ \\
\hline & CTG & 13 & $-0.06 \pm 0.05$ & & & & & \\
\hline
\end{tabular}

Values are presented an mean \pm standard deviation unless otherwise indicated.

PTCDG, progressive treadmill cognitive dual task gait training group; CTG, conventional treadmill gait training group; $d f$, degree of freedom; Cl, confidence interval.

${ }^{*} P<0.05$, statistical significance.

CTG group (change values, $-3.08 \%,-3.54 \%$, respectively) after intervention. Also, the swing and stance phase significantly increased in the PTCDG group (change values, $-1.47 \%,-1.47 \%$, respectively) than in the CTG group (change values, $-0.55 \%$, $-0.55 \%$, respectively) after intervention. Finally, the 10MWT showed a significant improvement in the PTCDG group (-0.16 $\mathrm{m} / \mathrm{sec}$ ) compared with the CTG group (-0.06 m/sec) (Table 4).

\section{DISCUSSION}

The main results of this study are as follows. The PTCDG group showed significantly better gait performance than the conventional treadmill gait training group. This study applied the same progressive treadmill cognitive dual-task gait training given in the previous studies (Silsupadol et al., 2006). The gait ability of the PTCDG group was significantly better than those of the CTG group after the treatment. In this study, the stride length and 
double support time of the affected side and the unaffected side were measured. The stride length tends to be shorter in the treadmill gait training than in the gait on the ground (Stolze et al., 1997).

Yang et al. (2007a) evaluated the velocity of 15 healthy adults, 15 patients with stroke patients with some difficulties in community gait, and 15 patients with chronic stroke patients capable of community gait with three motor tasks (i.e., simple gait, gait with buttoning up, and gait with a cup on a tray) and found that the gait associated with a dual-task was significantly faster than that not associated with a dual-task. Kizony et al. (2010) also reported that the dual cognition task increased the velocity after having twelve patients with stroke patients and ten healthy people walk on a treadmill at the velocity set by a subject and select products suitable for the situation at a virtual grocery store projected on a screen $1.5 \mathrm{~m}$ to the front of the subject. O'Shea et al. (2002) applied a cognitive task (addition and subtraction) to patients with Parkinson disease, who had decreased motor ability, while they were standing and found that the dual-task training improved their gait ability.

The cognitive-motor intervention occurs in patients with stroke patients who has lowered cognitive functions or gait disturbance because their ability to process cognitive and motor functions simultaneously decreases (Regnaux et al., 2005). It is believed that the gait ability of patients with stroke patients improved because cognitive and motor functions were simultaneously stimulated through the progressive treadmill cognitive dual-task gait training with supplementing these shortfalls.

Various studies have been conducted to evaluate the interaction between cognitive tasks with cognitive characteristics and motor task with motor characteristics (Paul et al., 2009). It is the most important to evaluate the motor function of patients with stroke patients reliably and accurately according to the cognitive task in order to evaluate the treatment plan and results (Wang et al., 2002). Cognitive dual-task training has been used widely in clinical practices. However, there are not enough studies comparing the differences between cognitive tasks. Moreover, the cognitive exercise of patients with stroke patients during gait has not been explained well and there are not enough studies on this topic.

The decreased cognitive function or the increased cognitive-motor intervention that occurs in patients with stroke patients is caused by the decreased ability to process cognitive and motor functions at the same time (Regnaux et al., 2005). A therapist must add cognitive and motor elements into the rehabilitation plan because the intervention between motor regulation ac- tivities while conducting two tasks (e.g., a cognitive task and gait) at the same time is an important therapeutic environmental element to consider for enhancing the functions of patients with neurological impairment independently (Haggard et al., 2000).

The results of this study showed that the PTCDG group had significantly different gait velocity, cadence, and single support times in the affected and unaffected side, affected swing phase, affected stance phase with the CTG group. However there was no significant difference between the groups in the stride lengths in the affected and unaffected sides.

Yang et al. (2007b) reported that the dual-task positively affected the walking of patients with chronic stroke patients. Kizony et al. (2010) evaluated the effects of the single task and the dual-task (e.g., velocity, stride length, and stride) using pre- and posttreatment comparison and found that both groups significantly improved velocity, stride length, and stride. Moreover, the treatment groups showed significantly faster velocity than the control group. Other studies also showed that cognitive tasks showed the same effect when the cognitive task training was applied to other subjects. O'Shea et al. (2002) applied a cognitive task (addition and subtraction) to patients with Parkinson disease, who had decreased motor ability, while they were standing. They evaluated gait characteristics such as velocity and stride length, and cognitive task performance. They found that it improved the cognitive task performance, reduced the error in task performance, and enhanced overall gait ability.

The cognitive-motor intervention occurs in patients with stroke patients who have lowered cognitive functions or gait distances and it is caused by the deteriorated ability to process cognitive and motor functions simultaneously (Regnaux et al., 2005). Regaining the mobility is the key to be independent in everyday life and the first goal of patients with stroke patients. Cognitive-motor training is considered to create a preparation step for the central nervous system that enables patients to effectively perform various tasks and motions (Barrett and Muzaffar, 2014). Previous studies reported that changing the gait pattern more or increasing the complexity of gait by proposing more dual-tasks activated the brain areas related to cognitive functions (Malouin et al., 2003, Yogev-Seligmann et al., 2008).

Independent gait is generally the ultimate rehabilitation goal for patients with stroke patients. This study evaluated the effects of the progressive treadmill cognitive dual-task gait training on the gait ability of patients with stroke patients. The progressive treadmill cognitive dual-task gait training improved the gait ability significantly more than the conventional treadmill gait train- 
ing. The results of this study indicated that the progressive treadmill cognitive dual-task gait training affected the cerebrum activation positively and effectively restored the gait ability.

The limitations of this study were that this study had a small sample size and could not confirm if the effects persisted because a follow-up study was not conducted. Therefore, future studies need to evaluate the effects of the treadmill gait training with utilizing the progressive cognitive dual-task on the rehabilitation of many patients with stroke patients. It will be also needed to evaluate the effects of the progressive treadmill cognitive dual-task gait training on the biomechanical factors such as muscle strength, energy consumption efficiency, muscle activity, and muscle fatigue.

In summary, this study demonstrated that the treadmill gait training with applying the progressive treadmill cognitive dual-task gait training was effective for enhancing the gait ability of patients with chronic stroke patients. It is believed that the program can be used as a training program for improving the gait ability of patients with chronic stroke patients who generally have difficulty in regaining the physical functions.

\section{CONFLCT OF INTEREST}

No potential conflict of interest relevant to this article was reported

\section{ACKNOWLEDGMENTS}

This work was supported by the Gimcheon University Grant of 2016.

\section{REFERENCES}

Barrett AM, Muzaffar T. Spatial cognitive rehabilitation and motor recovery after stroke. Curr Opin Neurol 2014;27:653-658.

Bowen A, Wenman R, Mickelborough J, Foster J, Hill E, Tallis R. Dualtask effects of talking while walking on velocity and balance following a stroke. Age Ageing 2001;30:319-323.

Campbell AJ, Borrie MJ, Spears GF, Jackson SL, Brown JS, Fitzgerald JL. Circumstances and consequences of falls experienced by a community population 70 years and over during a prospective study. Age Ageing 1990;19:136-141.

Dean CM, Richards CL, Malouin F. Walking speed over 10 metres overestimates locomotor capacity after stroke. Clin Rehabil 2001;15:415421.

Dennis A, Dawes H, Elsworth C, Collett J, Howells K, Wade DT, Izadi H,
Cockburn J. Fast walking under cognitive-motor interference conditions in chronic stroke. Brain Res 2009;1287:104-110.

Desmond DW, Moroney JT, Paik MC, Sano M, Mohr JP, Aboumatar S, Tseng CL, Chan S, Williams JB, Remien RH, Hauser WA, Stern Y. Frequency and clinical determinants of dementia after ischemic stroke. Neurology 2000;54:1124-1131.

Ditor DS, Macdonald MJ, Kamath MV, Bugaresti J, Adams M, McCartney $\mathrm{N}$, Hicks AL. The effects of body-weight supported treadmill training on cardiovascular regulation in individuals with motor-complete SCI. Spinal Cord 2005;43:664-673.

Evans JJ, Greenfield E, Wilson BA, Bateman A. Walking and talking therapy: improving cognitive-motor dual-tasking in neurological illness. J Int Neuropsychol Soc 2009;15:112-120.

Green J, Forster A, Young J. Reliability of gait speed measured by a timed walking test in patients one year after stroke. Clin Rehabil 2002;16:306314.

Haggard P, Cockburn J, Cock J, Fordham C, Wade D. Interference between gait and cognitive tasks in a rehabilitating neurological population. J Neurol Neurosurg Psychiatry 2000;69:479-486.

Hollman JH, Kovash FM, Kubik JJ, Linbo RA. Age-related differences in spatiotemporal markers of gait stability during dual task walking. Gait Posture 2007;26:113-119.

Kesar TM, Reisman DS, Perumal R, Jancosko AM, Higginson JS, Rudolph KS, Binder-Macleod SA. Combined effects of fast treadmill walking and functional electrical stimulation on post-stroke gait. Gait Posture 2011;33:309-313.

Kizony R, Levin MF, Hughey L, Perez C, Fung J. Cognitive load and dual-task performance during locomotion poststroke: a feasibility study using a functional virtual environment. Phys Ther 2010;90:252-260.

Malouin F, Richards CL, McFadyen B, Doyon J. New perspectives of locomotor rehabilitation after stroke. Med Sci (Paris) 2003;19:994-998.

Melzer I, Kurz I, Shahar D, Levi M, Oddsson L. Application of the voluntary step execution test to identify elderly fallers. Age Ageing 2007;36: 532-537.

O'Shea S, Morris ME, Iansek R. Dual task interference during gait in people with Parkinson disease: effects of motor versus cognitive secondary tasks. Phys Ther 2002;82:888-897.

Paul L, Ellis BM, Leese GP, McFadyen AK, McMurray B. The effect of a cognitive or motor task on gait parameters of diabetic patients, with and without neuropathy. Diabet Med 2009;26:234-239.

Pellecchia GL. Dual-task training reduces impact of cognitive task on postural sway. J Mot Behav 2005;37:239-246.

Regnaux JP, David D, Daniel O, Smail DB, Combeaud M, Bussel B. Evidence for cognitive processes involved in the control of steady state of walking in healthy subjects and after cerebral damage. Neurorehabil 
Neural Repair 2005;19:125-132.

Silsupadol P, Siu KC, Shumway-Cook A, Woollacott MH. Training of balance under single- and dual-task conditions in older adults with balance impairment. Phys Ther 2006;86:269-281.

Stolze H, Kuhtz-Buschbeck JP, Mondwurf C, Boczek-Funcke A, Jöhnk K, Deuschl G, Illert M. Gait analysis during treadmill and overground locomotion in children and adults. Electroencephalogr Clin Neurophysiol 1997;105:490-497.

van Uden CJ, Besser MP. Test-retest reliability of temporal and spatial gait characteristics measured with an instrumented walkway system (GAITRite). BMC Musculoskelet Disord 2004;5:13.
Yang YR, Chen YC, Lee CS, Cheng SJ, Wang RY. Dual-task-related gait changes in individuals with stroke. Gait Posture 2007a;25:185-190.

Yang YR, Wang RY, Chen YC, Kao MJ. Dual-task exercise improves walking ability in chronic stroke: a randomized controlled trial. Arch Phys Med Rehabil 2007b;88:1236-1240.

Yogev-Seligmann G, Hausdorff JM, Giladi N. The role of executive function and attention in gait. Mov Disord 2008;23:329-342.

Wang CH, Hsieh CL, Dai MH, Chen CH, Lai YF. Inter-rater reliability and validity of the stroke rehabilitation assessment of movement (stream) instrument. J Rehabil Med 2002;34:20-24. 